\title{
A Comparison of Unimodal and Multimodal Measurements of Driver Stress in Real-World Driving Conditions
}

\author{
Christine Spencer \\ cspencer03@qub.ac.uk \\ Queen's University Belfast \\ Belfast, Antrim \\ Alex Lee \\ alex.lee@volvocars.com \\ Volvo Cars USA \\ Sunnyvale, California \\ Maria Iozzo \\ maria.iozzo@volvocars.com \\ Volvo Cars USA \\ Sunnyvale, California
}

\author{
İbrahim Alper Koç \\ iakoc@sensum.co \\ Sensum \\ Belfast, Antrim
}

\author{
Anupama Mahesh Dhareshwar \\ anupama.dhareshwar@volvocars.com \\ Volvo Cars \\ Gothenburg, Västra Götaland
}

\author{
Gawain Morrison \\ gawain@sensum.co \\ Sensum \\ Belfast, Antrim
}

\author{
Chihiro Suga \\ chihiro.suga@volvocars.com \\ Volvo Cars USA \\ Sunnyvale, California \\ Elin Franzén \\ elin.franzen@volvocars.com \\ Volvo Cars \\ Gothenburg, Västra Götaland \\ Gary McKeown \\ g.mckeown@qub.ac.uk \\ Queen's University Belfast \\ Belfast, Antrim
}

\begin{abstract}
Objectively measuring drivers' emotions in real-world conditions is a challenging endeavour. This study investigated whether drivers' emotional responses were captured more meaningfully by unimodal measurements or by a multimodal machine-learning approach. Ten participants drove a 23-mile route around Sunnyvale, California, while their heart rate, breathing rate and facial expressions were recorded. At regular intervals, participants indicated how they were feeling. After the study, independent observers reviewed a sample of the videotaped sessions, classifying the participants as experiencing high or low levels of stress according to their behaviour. The degree to which drivers' self-report scores, single physiological data streams and facial behaviour-as judged by a facial recognition machine classifier-reflected their actual stress levels was compared to the multimodal algorithmic estimates which combined physiological and facial data outputs. The results showed that, compared to the other single data modes, the multimodal approach captured how the participants were feeling in a way which most meaningfully corresponded with their observed behaviour. The findings re-affirm the need for multimodal emotion-recognition systems for capturing driver stress. The limitations of the study are also discussed.
\end{abstract}

\section{CCS CONCEPTS}

- Applied computing $\rightarrow$ Psychology; $\bullet$ Human-centered computing $\rightarrow$ User studies; • Computing methodologies $\rightarrow$ Supervised learning by classification.

Permission to make digital or hard copies of all or part of this work for personal or classroom use is granted without fee provided that copies are not made or distributed for profit or commercial advantage and that copies bear this notice and the full citation on the first page. Copyrights for components of this work owned by others than ACM must be honored. Abstracting with credit is permitted. To copy otherwise, or republish, to post on servers or to redistribute to lists, requires prior specific permission and/or a fee. Request permissions from permissions@acm.org.

Conference'17, July 2017, Washington, DC, USA

(C) 2020 Association for Computing Machinery.

ACM ISBN 978-x-xxxx-xxxx-x/YY/MM...\$15.00

https://doi.org/10.1145/nnnnnnn.nnnnnnn

\section{KEYWORDS}

multimodal stress detection, real-world driving

\section{ACM Reference Format:}

Christine Spencer, İbrahim Alper Koç, Chihiro Suga, Alex Lee, Anupama Mahesh Dhareshwar, Elin Franzén, Maria Iozzo, Gawain Morrison, and Gary McKeown. 2020. A Comparison of Unimodal and Multimodal Measurements of Driver Stress in Real-World Driving Conditions. In Proceedings of ACM Conference (Conference'17). ACM, New York, NY, USA, 10 pages. https: //doi.org/10.1145/nnnnnnn.nnnnnnn

\section{INTRODUCTION}

The ability to meaningfully capture drivers' stress levels in realworld conditions has important applications for maintaining driver health, safety and performance, as well for the development of empathic vehicular feedback mechanisms for driver stress reduction-a thriving research area [1][2][3]. The experience of psychological stress-a state of emotional or mental tension that people experience in response to taxing or adverse environmental demands or events which threaten their well-being [4]-is not only harmful to health [5][6][7][8], but is also known to be a predictor of risky driving behaviour [9] and the incidence of traffic accidents [10]. Experiencing stress is thought to adversely impact an individual's cognitive flexibility [11], working memory [12][13] as well as navigation [14] and decision-making abilities [15]-critical factors for safe and careful driving performance. Evidence from several simulator driving studies has shown that people tend to demonstrate impaired performance when driving under stressful conditions [16][17][18], such as the experience of unpleasantly high levels of cognitive load [19].

Additionally, as higher numbers of users gradually avail of assisted and automated driving technologies, it is also becoming increasingly important to accurately monitor drivers' emotions as they engage in shared control with fully or partially automated driving systems. Drivers may experience stress during this interaction, as the human-machine interfaces that facilitate the exchange continue to change in appearance and interaction requirements [20]. 
People's trust in this type of technology may vary[21][22]-drivers with low levels of trust may remain in an unpleasant, tense state from the point of relinquishing control right up until the point of regaining control of their vehicle. This situation is not ideal as the experience of stress can impair drivers' ability to retrieve control from the automated system [23][24], may cause non-optimal manual driving performance, and may even result in a reduced tendency to engage with the assisted system in the future.

Driving performance is thought to deteriorate when drivers experience emotional extremes-becoming impaired at low, as well as high, levels of cognitive load [25][26]. Some drivers may, for example, over trust an assisted driving system and not pay attention to the road when the vehicle is in autonomous mode. They may even engage in irresponsible relaxation activities, such as napping, during this time. In situations where manual takeover is still necessary [27], such behaviours could have life-threatening consequences. The ideal driver should remain adequately engaged and calm, ready to resume control if required. It is critical to maintain appropriate levels of driver stress-whereby an individual's comfort is maximised yet they are still able to safely operate the takeover. Establishing an effective and reliable means of measuring drivers' emotions presents a complex methodological challenge, but is critical for enhancing driver safety and user experience.

\section{PREVIOUS LITERATURE}

2.0.1 Defining emotions. Emotions are not easily defined but are generally thought to be pleasant or unpleasant subjective experiences that people have in response to environmental stimuli, involving changes in physiology and behaviour [28]. Following Russell's Circumplex Model of Affect [29], it is possible to estimate a person's emotional state in terms of two dimensions-valence and arousal. Valence refers to the subjective quality of an emotional experience-whether a person feels positive or negative-while arousal refers to the degree to which an individual's physiological state is activated and ready to respond to cope with environmental demands. Stress is characterised by high levels of arousal and negative valence, and can be theoretically represented in Quadrant 1 of a two-dimensional valence-arousal space, as illustrated in Figure 1. Lower-arousal states are represented in the lower quadrants-for example, calmness would be represented in Quadrant 3 while less positive low-arousal states such as boredom, or fatigue, would be represented in Quadrant 4.

\subsection{Measurement approaches}

Estimating drivers' valence and arousal levels to place them within these theoretical zones presents a complex challenge.

2.1.1 Drivers' self-report. The most commonly-used approach seems to be the use of self-report, where drivers are asked to report how they are feeling, either during, or after, the drive. A disadvantage of this approach is that it may be difficult for people to objectively gauge their own feelings or to judge their physiological activation levels. Standardisation can be an issue, as people may not necessarily understand and conceptualise emotions in the same way [30]. Additionally, people may vary in their scoring tendencies, with some individuals scoring themselves more liberally compared to others [31]. Self-report measures can be affected by

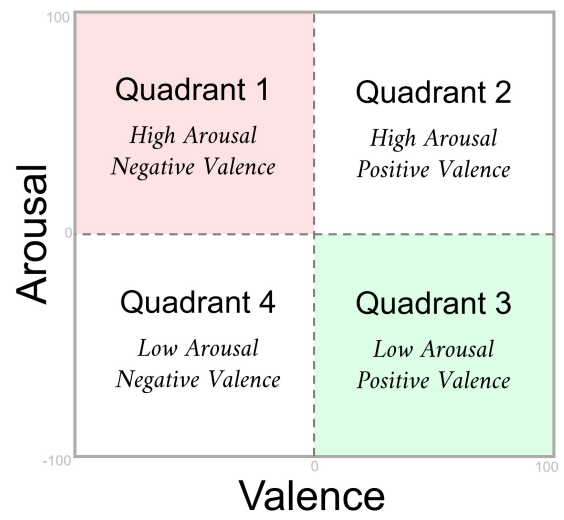

Figure 1: Theoretical valence-arousal space

social desirability bias [32][33] - participants in a user study may feel obliged to report favourable feelings towards a driving system to not appear critical or rude, for example. Additionally, the act of reporting during the study disrupts the flow of the participant's driving experience, and can even have an immediate impact on a person's physiological state [34]. Perhaps most pressingly, emotions are dynamic phenomena-an individual's arousal and valence levels can change quickly and dynamically [35][36]. The use of self-report scoring does not allow for continuous measurement and so may not adequately capture how an individual's emotional experience evolves over time.

2.1.2 Drivers' physiological signals. The practice of analysing changes in drivers' physiological signals may offer a more objective means of estimating how they are feeling. The experience of stress is thought to be characterised by elevation in heart rate [37][38][39], breathing rate [40][41][42] and skin conductance response [43][44][31][45][46]. This approach has been employed in both real [47][43][48] and simulated [44][49] driving studies. However, the quality of cardiac data can be affected by movement artefacts [50] while skin conductance signals can be sensitive to temperature, humidity or participant age [51]. Additionally, it has been questioned whether physiological changes are always experienced as specific emotions, or whether such changes necessarily impact behaviour [52][53]. The effect of stressful events on a person's physiology can be long-lasting, while the anticipation of stressful events which are yet to happen can also heighten a person's level of arousal in advance-making interpretation of physiological changes complex [54]. Additionally, some wearable physiological sensors may not be well-suited for real-driving situations, such as the placement of electrodes on participants' fingertips to measure skin conductance activity.

2.1.3 Drivers' facial expressions. Facial cues offer a noninvasive method of estimating drivers' emotions [55][56]. However, the notion that facial expressions dependably convey specific emotions is controversial [57][58][59]. Nonverbal signals can have different meanings across contexts [60][61]. For example, smiling behaviour can indicate amusement, but can also indicate derision [62], social discomfort [63] or embarrassment [28]. There are other factors to consider-perhaps as a result of societal expectations 
[64][65], it has been suggested that men do not always express their emotions as openly as women [66], although this is contentious [67] and likely depends on context [68]. Additionally, facial expressions are thought to be tools that people utilise to let others know how they are feeling [69]-in situations when drivers are alone, they may display fewer facial expressions as there is no one present with whom they can communicate how they are feeling.

2.1.4 Multimodal approach. As each of the mentioned approaches to analysing drivers' emotions-the analysis of self-report, physiological signals or facial expressions-is associated with certain caveats, it is preferable to estimate drivers' valence and arousal levels by using a multimodal approach. There is an increasing movement towards the use of multimodal emotion-prediction systems [70][71][72], with a meta-analysis reporting that unimodal systems are outperformed $85 \%$ of the time by multimodal systems for both natural versus acted emotions [73]. Such approaches allow for the inherent multidimensional nature of emotions and the context-dependent nature of their expression-that in some situations, a person may not reveal their emotional state outwardly, in which case their physiological responses might offer the best insight into how they are feeling. In more social settings, where facial expressions come to the fore as tools for social communication [74], a person's heart and breathing rate might indicate a high level of physiological activation, while facial cues may provide the strongest indication of valence. By combining physiological signals with facial behaviour using a data-fusion approach, it is theoretically possible to build a richer and more meaningful picture of how a person is feeling.

\subsection{Study overview}

The present study compared how meaningfully drivers' emotional responses were captured by single data streams compared to a multimodal approach. The comparison analysis was performed using sample data from a driving study where participants drove for 45 minutes on actual highways and industrial roads in Sunnyvale, California. Participants' physiology and facial behaviour were captured as they completed periods of manual driving as well as periods where they interacted with an assisted driving system. The degree to which drivers' self-report scores, single physiological data streams and facial behaviour reflected their actual stress levels-as classified by observer judgments of their behaviour-was compared to multimodal algorithmic estimates which fused physiological and facial data outputs.

\section{METHOD}

\subsection{Participants}

In the original study, 36 employees within a leading international automotive brand were recruited for an internal study to test user reactions to an assisted driving system. As the footage from each participant's session had to be reviewed in-depth by two research assistants, this was a highly time-consuming process and it was therefore only possible to analyse $30 \%$ of the sample here. Datarecording difficulties meant that data for 3 participants was not usable. From the remaining sample of 33, session data was extracted for 10 randomly-selected individuals ( 3 females and 7 males, mean age $=38.8$ years). Participants were all at least 18 years old, possessed a full driving licence, drove a minimum of three to five days a week on average and had moderate experience of using the brand's assisted driving functionality.

\subsection{Materials and measures}

3.2.1 Questionnaires. Participants completed two questionnaires-one before and one after their drive-answering questions about their personality traits, driving tendencies, as well as their attitude towards the assisted driving system that they tested and towards new technology in general. These responses are not analysed here.

3.2.2 Self-report scores. At various checkpoints during the drive, the experimenter asked the participant to verbally state how positive they were feeling on a scale of 1 to 10 , with 1 being "not at all positive" to 10 being "highly positive". The experimenter recorded their response on a mobile device. The scores were averaged to obtain a mean self-report positivity score for each participant's session-a low score representing higher levels of stress.

3.2.3 Physiological signals. Each participant wore an Equivital EQ02+ LifeMonitor. This high-quality [75][76][77] sensor belt is made of a breathable, light-weight fabric that makes direct contact with the upper body. It has in-built sensors for measuring heart and breathing rate, and a skin conductance response attachment-a pair of electrodes which are placed on the underside of two fingers to record changes in sweat gland activity. Data is stored in the Electronics Module (SEM) unit-docked inside the vest. The placement of these sensors is illustrated in Figure 2.

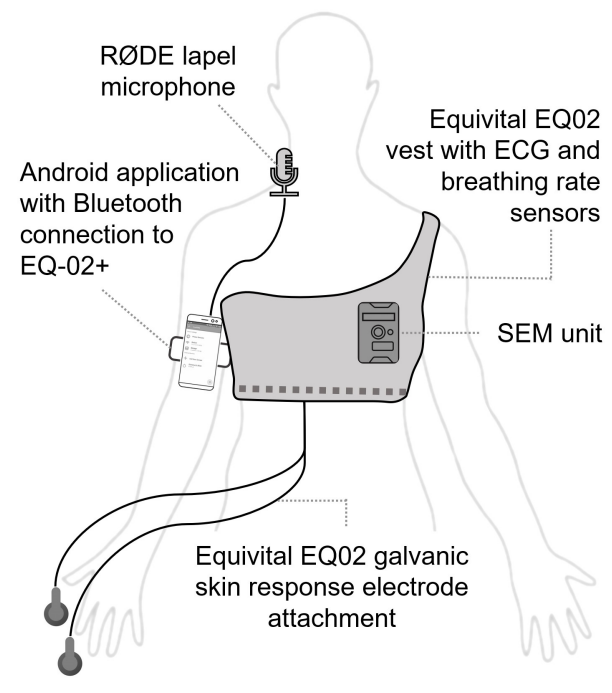

Figure 2: Placement of wearable sensors

3.2.4 Facial behaviour. Participants' facial expressions were recorded with a dash-mounted ELP Webcam USB 1080P Camera. The camera was placed in the left-bottom corner of the windscreen, angled upwards to capture the participant's facial movements 
as directly as possible. This camera, which works during both daytime and night-time conditions, allowed high-quality recording to continue regardless of any disruptions in lighting during the drive-for example, in shaded areas. The recorded facial expression footage was analysed in real-time using the SHORE [78] facial classification library. For each frame of footage, a participant's emotional expression was classified according to several discrete emotion categories, applying a confidence score for each category. In the current case, only SHORE's scores for "Positive Valence" were used-this score was presumed to reflect the degree to which the person was demonstrating positive facial behaviours, such as smiling and laughter.

3.2.5 Context data. Context information-the driver's view of the road-was recorded with a Logitech RGB webcam camera which was mounted mid-way on the dash, pointing outwards. This captured any emotionally-salient events encountered during the drive which may have had an impact on the driver's stress levels, such as the behaviour of other drivers or traffic congestion. Anything the participants said, or any nonverbal vocalisations they produced, were recorded with a RØDE lapel microphone. The placement of these devices is illustrated in Figure 3.

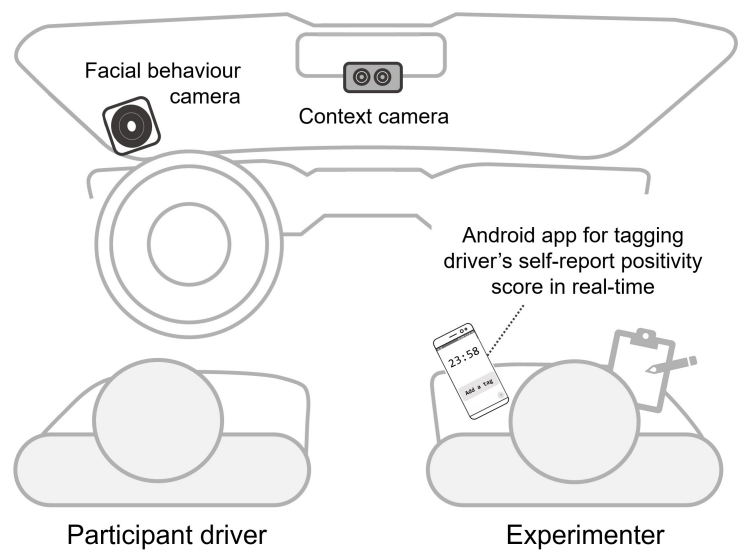

Figure 3: Placement of data-capture devices within cabin

3.2.6 Multimodal predictions. Data from the cameras, EQ02 vest and the experimenter's mobile were transmitted via Bluetooth to a Synsis ${ }^{\text {TM}}$ Empathic AI Developer Kit developed by Sensum [79]. Data capture was initiated when the experimenter hit the "Start Session" button on the Synsis user interface, allowing all data streams to be time-synchronised, regardless of format and sampling frequency. Participants' emotions were then predicted by the kit's multimodal arousal and valence algorithms. These were developed using a decision-tree machine learning approach-using data collected in previous studies where people were required to complete stressful and calming activities in a simulated driving context. This data was labelled by multiple annotators using a continuous scoring method. The models were then trained to recognise changes in physiological activation and "positivity" or "negativity" based on these annotated labels. Approximately one valence and one arousal prediction score were produced per second for each participant's session.

\subsection{Procedure}

3.3.1 Driving task. Participants were fitted with the wearable sensors, before receiving a brief explanation of the study. They then drove a 23-mile route around Sunnyvale, California, which took approximately 40-45 minutes on average. The driving task began on a brief section of industrial road. Participants then entered a long section of highway driving which involved a roundabout and periods of using the assisted driving system. This was followed by a brief section of industrial roads again and a few minutes in a more pedestrianised downtown area. Participants then returned to the testing area. The route is summarised in Figure 4.

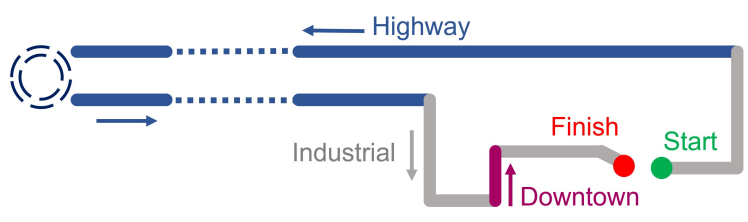

Figure 4: Route driven by participants

3.3.2 Behavioural stress classification. After the study, two undergraduate research assistants, who were blind to the study aims and protocol, were asked to review the videotaped footage of each participant as well as the corresponding context footage of the road and behaviour of other drivers. The assistants were independently asked to classify which individuals seemed to be experiencing higher levels of stress based on their behaviour during the drive. The assistants' classifications were in agreement in each instance-both perceived five of the drivers to be demonstrating stressed behaviour. These individuals were allocated to the "High Stress Behaviour" group. The remaining five drivers were perceived to be calmer, and were allocated to the "Low Stress Behaviour" group.

\subsection{Results}

3.4.1 Self-report scores. Participants' verbally self-reported positivity scores were compared. Each group's mean score is shown in Table 1. This result indicated that participants in both groups reported feeling generally positive-there was very little difference between them.

Table 1: Mean Verbally Self-reported Positivity Scores

\begin{tabular}{ccc}
\hline Group & Mean & SD \\
\hline "High Stress Behaviour" & 6.91 & 2.64 \\
"Low Stress Behaviour" & 6.80 & 2.09 \\
\hline
\end{tabular}

3.4.2 Generalised additive models. The changes in participants' facial behaviour, physiological signals and multimodal arousal and valence predictions were examined over time using generalised additive models (GAMs) with restricted maximum 
likelihood estimation. This method is preferred over standard regression for analysing non-linear trends[80]. The GAMs were created in $\mathrm{R}$ [81] using the mgcv package [82] and took the generalised form:

$$
y=\alpha+f(\text { time })+\epsilon \sim N\left(0, \sigma^{2}\right)
$$

where $y$ represents either breathing rate, heart rate, facial positivity, arousal or valence, $\alpha$ represents the intercept, $f$ (time) represents the smoothing function, and $\epsilon$ represents normally distributed errors. The best way to interpret GAMs is through graphical presentation of the smooths [83]. Visualisations were performed in $\mathrm{R}$ using ggplot2 [84] and tidymv [85]. The grey variability bands surrounding each smooth represent pointwise $95 \%$ confidence intervals. Where they overlap there is no significant difference between the groups. Due to the scaling of each graph, these bands are only wide enough to be visibly observable in Figure 9.

3.4.3 Non-linear data preparation. GAMs were created for breathing rate, heart rate and facial positivity, as well as for the multimodal valence and arousal predictions. Before GAMs could be created, it was necessary to prepare the data and check its suitability for this form of modelling. GPS location tags were used to trim the different sessions to the same length so that the journeys were as comparable as possible. The gam.check function in the mgcv package was used to check for independence of model residuals for each data type. Only data for skin conductance did not pass this check and is therefore not analysed here. Autocorrelation may have been a problem, or it is also possible that, as this is a highly sensitive signal [86], there may have been a higher degree of inter-individual variation which prevented GAMs from being produced-a larger participant sample might have resolved this issue.

3.4.4 Breathing rate. Separate GAMs were created to model changes in each group's breathing rate over time. The smooths for both the "High" (edf $=5.96, F=7.70, p<.001)$ and the "Low" $(e d f=2.80, F=9.09, p<.001)$ stress groups were significant. As shown in Figure 5, the "High" stress group's breathing rate tended to be consistently higher than the "Low" group's across the session. The confidence intervals do not overlap-indicating that these two groups' breathing rates were significantly different across the session.

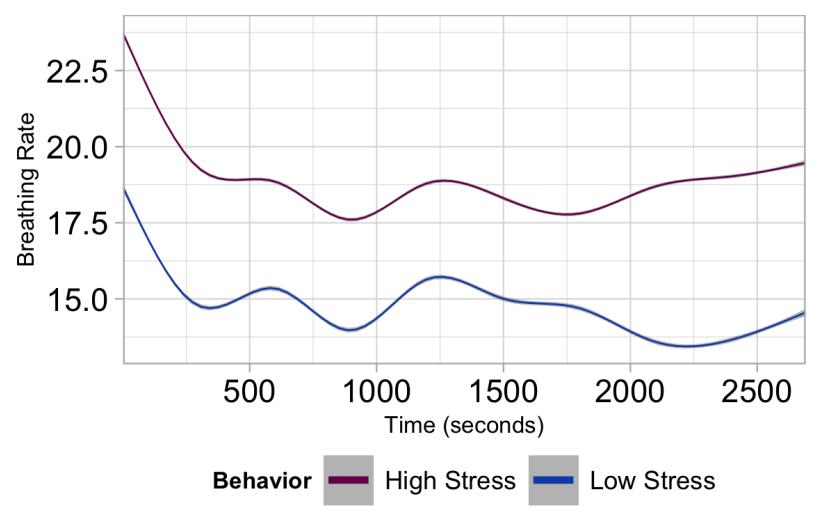

Figure 5: Changes in each group's breathing rate
3.4.5 Heart rate. Separate GAMs modelled changes in each group's heart rate over time. The GAM smooths for both the "High" $(e d f=2.80, F=18.48, p<.001)$ and "Low" ( $e d f=3.80$, $F=12.53, p<.001)$ stress groups were significant. As shown in Figure 6 , both groups' heart rates were relatively high at the beginning of the session, gradually decreasing as time went on. However, participants who displayed more stressful behaviour showed a lesser rate of deceleration compared to the "Low" stress group. For the majority of the session, the confidence intervals do not overlap, indicating that the two group's heart rates were significantly different. However, at around 750-1500 seconds, when the participants were using the assisted driving system, the two groups' heart rates were almost equivalent.

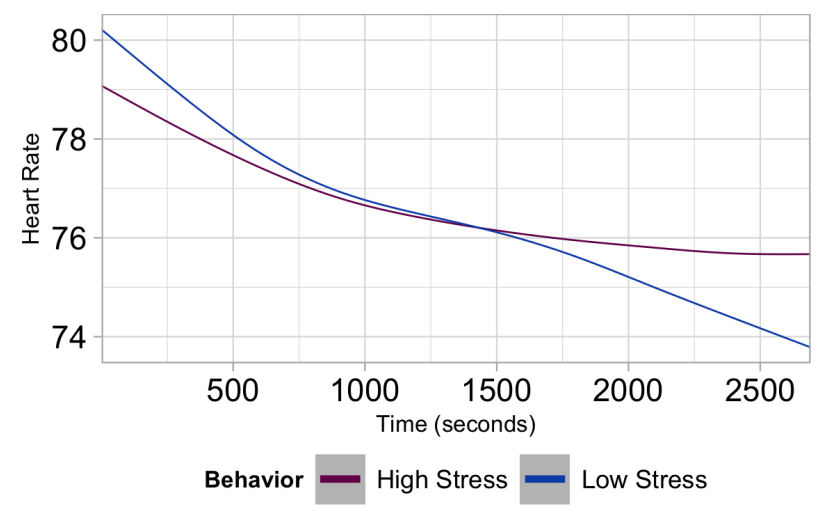

Figure 6: Changes in each group's heart rate

3.4.6 Facial behaviour. The same approach was used to model changes in participants' facial positivity levels over time. The smooths were significant for both the "High" (edf $=8.90, F=$ $147.90, p<.001)$ and "Low" ( $e d f=8.93, F=113.80, p<.001)$ stress groups. As shown in Figure 7, participants' facial behaviours were generally negatively-valenced for both groups across the session. However, the "High" stress participants were consistently more positive by around ten points across the session. As demonstrated by a lack of overlap between each smooth's confidence intervals, the differences between the groups were significant.

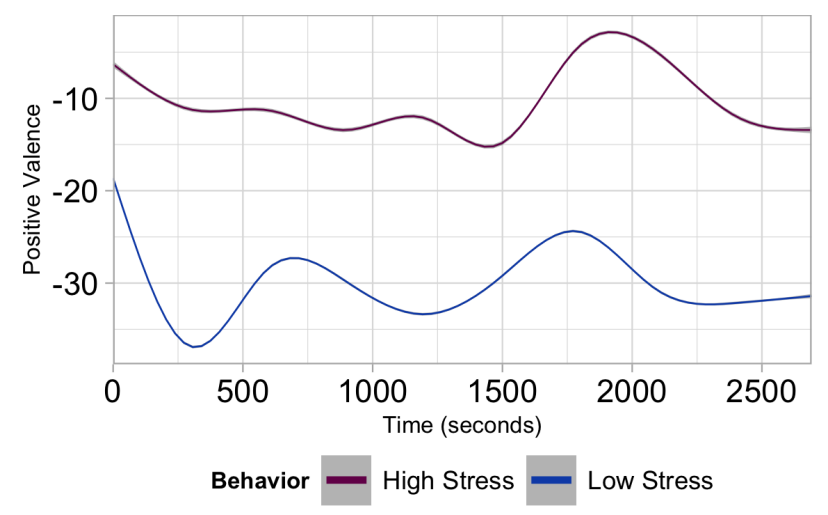

Figure 7: Changes in each group's facial positivity levels 
3.4.7 Multimodal arousal predictions. Separate GAMs were created to model changes in each group's arousal levels over time. The smooths were significant for both the "High" (edf $=8.65, F=$ $27.04, p<.001)$ and "Low" ( $e d f=8.85, F=54.18, p<.001)$ stress groups. As shown in Figure 8, participants who behaved in a more stressed way had consistently higher levels of arousal across the session compared to the "Low" stress group. As shown by the separation of confidence intervals, this difference was significant.

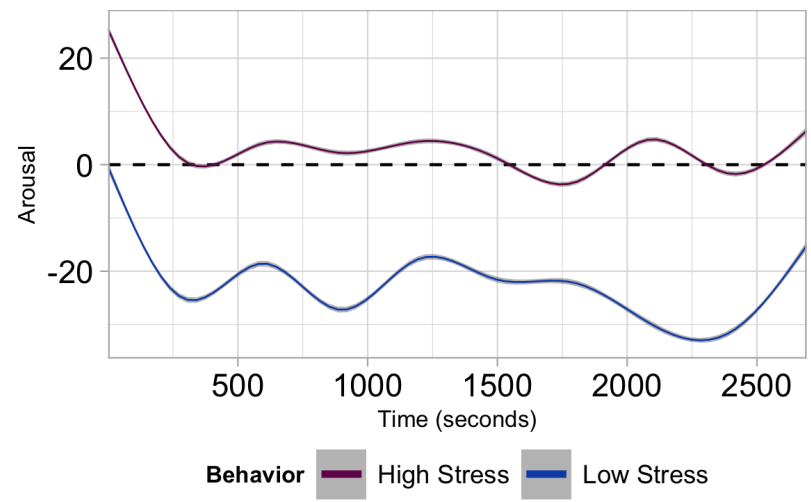

Figure 8: Changes in each group's arousal levels

3.4.8 Multimodal valence predictions. Separate GAMs were created to model changes in each group's valence levels over the session. The smooths were significant for both the "High" ( $e d f=$ 8.79, $F=65.64, p<.001)$ and "Low" $(e d f=8.88, F=74.48, p<.001)$ stress groups. As shown in Figure 9, participants who demonstrated more stressful behaviour experienced greater levels of negative valence across the session compared to the "Low" stress group. While the "Low" stress participants became gradually more positive, this pattern did not occur for the "High" stress individuals. For the majority of the session, this difference was significant-indicated by the non-overlapping confidence intervals which are wider and more clearly visible in this case, compared to the other data streams. However, when participants were using the assisted driving system-approximately between 600 and 1000 seconds into the session-the two groups' valence levels became more aligned, and were practically equivalent at the points where the confidence intervals overlap.

3.4.9 Combined valence-arousal predictions. Figure 10 is a visualisation of all the arousal and valence predictions produced for each group across all sessions. The predictions for the "High" stress group were most densely populated in Quadrant 1-a zone in the VA space associated with stress. Conversely, the predictions for participants who behaved in a less stressed way were more heavily concentrated in Quadrant 3-an area theoretically associated with relaxation and calm.

\section{DISCUSSION}

The current study compared different methods of measuring driver stress levels-assessing whether participants' emotional responses were reflected more meaningfully by their verbal self-report scores,

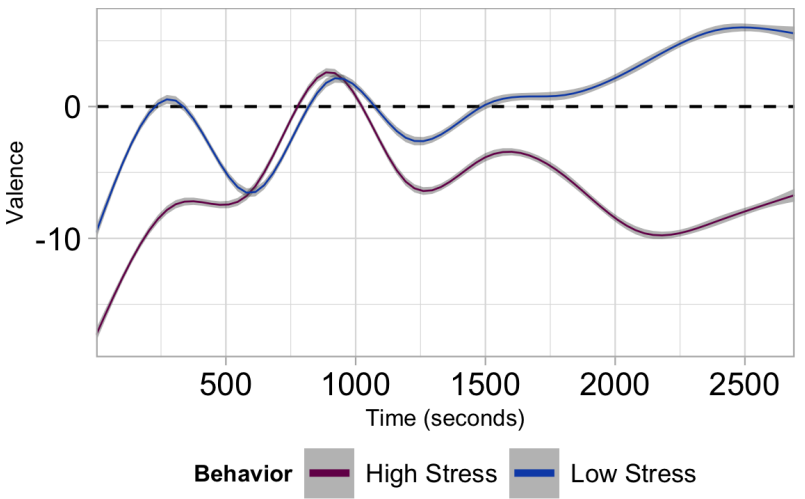

Figure 9: Changes in each group's valence levels

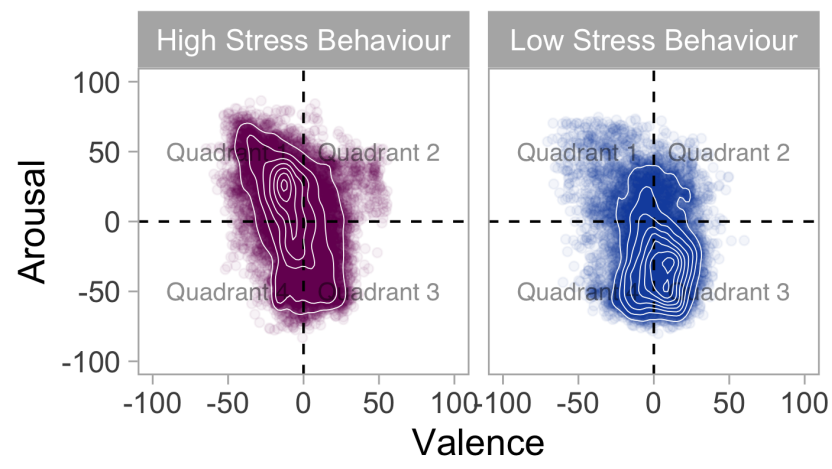

The white contour lines demarcate zones in the VA space where the valence-arousal predictions are populated to the same degree. The innermost "peak" represents the emotional zone most experienced by participants in each group.

Figure 10: Valence-arousal space predictions for each group

by their raw physiological signals, their facial expressions or by a multimodal algorithmic prediction of their arousal and valence levels. The results showed that the multimodal arousal and valence predictions corresponded correctly with the observers' judgments of the participants' stress levels. The multimodal approach particularly outperformed the self-report approach, as the drivers' self-report scores were not associated particularly meaningfully with their behaviour. Although the independent observers judged one group of participants to be demonstrating greater levels of stress compared with the other group, there was relatively little difference between the groups' mean positivity scores. Therefore, the "High" stress participants reported themselves to be just as positive as the participants who seemed to be visibly calmer. Additionally, although the two groups reported feeling relatively similar levels of stress, there were clear differences between their physiological signals. The participants who showed more stressful behaviours had a consistently higher breathing rate across the session. They also did not experience the same level of heart rate deceleration as participants in the "Low" stress group.

However, these results highlight the nuances involved in interpreting how a person is feeling from a single data stream. While there was a clear distinction between the groups' breathing rate 
patterns in Figure 5, the distinction is less clear in Figure 6 where, at one stage, the groups' heart rates were roughly equivalent. In the case of facial behaviour, although there was a clear difference between the two groups, it was not a simple case of the stressed participants displaying greater negativity. Participants who were perceived to be more stressed consistently displayed greater levels of "positive" facial behaviours compared to those who were perceived to be calmer. It is possible that these participants may have laughed and smiled as a means of coping with the stressful situation or to appear calm to the experimenter. It is possible that participants in the "Low" stress group may have demonstrated a combination of occasional frowning in the absence of intense smiling-resulting in a more "negative" score. This finding illustrates why it is important to interpret the output of facial expression classifiers carefully, in the context of other sources of evidence about an individual's state, where possible.

Here, only participants' facial "positivity" scores were analysed as they fluctuated over time. However, information about the timing of each positive behaviour was not taken into account. Nonverbal expressions work together in subtle ways to convey complex social messages [87]-it may have been more useful to explore the frequency with which these positive behaviours occurred. It is possible that it was the volatility of the SHORE Positive Valence signal which was more important-perhaps negative valence is not characterised by the absence of smiling and laughter, but is rather signalled by frequent, yet erratic and brief bursts of these signals. A future investigation could explore whether including the volatility of participants' facial positivity scores as a data input improves the performance of the multimodal predictions.

There are some limitations to the current approach that must be considered. Comparisons were performed for a relatively small number of individuals-only $30 \%$ of the original participant sample were analysed due to resource-constraints. The in-depth post-study review of each participant's videotaped face camera and context footage, performed by two assistants, took at least 2 hours for each participant. There was therefore insufficient time to classify the entire sample in the same way. However, although only ten individuals were examined here, each session produced-on average-536.9 heart rate, 179 breathing rate and 18430.6 facial positivity values, as well as 2571.5 multimodal valence and 2569.9 arousal predictions. Therefore, the results should be considered in light of the large volume of data points recorded as well as the length of each session-rich, complex, multimodal real-world driving data was captured for at least 40 minutes for every participant. It should also be acknowledged that the current analyses focused on three specific data inputs. Alongside skin conductance, which was captured but not analysed here, there are many other important data inputs that can be used to provide an even richer picture of a person's stress level, such as heart rate variability [88] or changes in skin temperature [89]. Gesture and body movements [90][91], as well as acoustic and speech signals [92][93][94], can also provide meaningful insights about how an individual is feeling.

Participants' stress levels were classified as high or low by human judges-a future study could also incorporate metrics of driver performance such as drivers' speed [95] or braking tendencies [96][97]. The current approach would also have been stronger with the addition of context data inputs. The behaviour of other drivers and the navigation of heavy traffic are known to trigger stressful events [98], and capturing these, along with information about a drivers' acceleration, speed, braking and gas usage [99][1] would add additional information by which algorithms can make meaningful predictions about how a person is likely to be feeling in that context. It is also appreciated that the current procedure required participants to wear a vest that made direct contact with their skin, as well as electrodes on their fingers-this may not always be possible in normal driving conditions. However, increasingly reliable non-invasive methods of capturing physiological changes continue to be developed, such as the presence of skin conductance electrodes on the steering wheel [1], heart rate detection by ultra-wideband impulse radar technology [100], the use of cameras to capture pulse rate [101][102], heart rate variability [103][104] as well as head motion [102]. Machine vision systems are being developed to track cardio-respiratory changes from video for more than one person at a time with sophisticated noise removal [105]. As individuals tend to exert greater hand pressure under stressful conditions [106], it may also be possible to detect driver stress by monitoring changes in hand grip pressure on the steering wheel and in the use of in-vehicle interfaces [1].

In conclusion, the current findings reaffirm the importance of measuring driver stress levels using multiple data modes. By using machine learning models which are sensitive to subtle changes in physiological signals in conjunction with patterns of facial behaviour, the multimodal approach produced emotion predictions which corresponded with the drivers' observed behaviour in a way that was more meaningful than simply assessing their physiological signals and facial behaviour in isolation, or by asking them to judge how they were feeling. As we move towards progressively non-invasive in-cabin physiological sensing techniques, as well as towards increasingly automated context event labelling technology, data obtained from further studies with more participants will help to train increasingly sophisticated, context-aware, multimodal valence-arousal prediction algorithms.

\section{ACKNOWLEDGMENTS}

Thank you to all the participants who took part in this study.

\section{REFERENCES}

[1] Javier Hernandez, Daniel McDuff, Xavier Benavides, Judith Amores, Pattie Maes, and Rosalind Picard. Autoemotive: Bringing empathy to the driving experience to manage stress. In Proceedings of the 2014 Companion Publication on Designing Interactive Systems, DIS Companion '14, page 53-56, New York, NY, USA, 2014. Association for Computing Machinery.

[2] Sarah Theres Völkel, Julia Graefe, Ramona Schödel, Renate Häuslschmid, Clemens Stachl, Quay Au, and Heinrich Hussmann. I drive my car and my states drive me: Visualizing driver's emotional and physical states. In Adjunct Proceedings of the 10th International Conference on Automotive User Interfaces and Interactive Vehicular Applications, AutomotiveUI '18, page 198-203, New York, NY, USA, 2018. Association for Computing Machinery.

[3] Michael Braun, Florian Weber, and Florian Alt. Affective automotive user interfaces - reviewing the state of emotion regulation in the car. arXiv preprint arXiv:2003.13731, 032020.

[4] Susan Folkman. Stress: Appraisal and Coping, pages 1913-1915. Springer New York, New York, NY, 2013.

[5] Suzanne C Segerstrom and Gregory E Miller. Psychological stress and the human immune system: a meta-analytic study of 30 years of inquiry. Psychological bulletin, 130(4):601, 2004.

[6] Jos F Brosschot, William Gerin, and Julian F Thayer. The perseverative cognition hypothesis: A review of worry, prolonged stress-related physiological activation, and health. Fournal of psychosomatic research, 60(2):113-124, 2006 
[7] Cristina Ottaviani, Julian F Thayer, Bart Verkuil, Antonia Lonigro, Barbara Medea, Alessandro Couyoumdjian, and Jos F Brosschot. Physiological concomitants of perseverative cognition: A systematic review and meta-analysis Psychological Bulletin, 142(3):231, 2016.

[8] Audrey Blanc-Lapierre, Marie-Claude Rousseau, and Marie-Elise Parent. Perceived workplace stress is associated with an increased risk of prostate cancer before age 65. Frontiers in oncology, 7:269, 2017.

[9] Corneliu-Eugen Havârneanu, Cornelia Măirean, and Simona-Andreea Popuşoi. Workplace stress as predictor of risky driving behavior among taxi drivers. the role of job-related affective state and taxi driving experience. Safety science, 111:264-270, 2019

[10] Sue Cartwright, Cary L Cooper, and Andrea Barron. The company car driver, occupational stress as a predictor of motor vehicle accident involvement. Human Relations, 49(2):195-208, 1996

[11] Grant S Shields, Matthew A Sazma, and Andrew P Yonelinas. The effects of acute stress on core executive functions: A meta-analysis and comparison with cortisol. Neuroscience \& Biobehavioral Reviews, 68:651-668, 2016.

[12] Nicole YL Oei, Walter TAM Everaerd, Bernet M Elzinga, Sonja van Well, and Bob Bermond. Psychosocial stress impairs working memory at high loads: an association with cortisol levels and memory retrieval. Stress, 9(3):133-141, 2006

[13] Oriel FeldmanHall, Candace M Raio, Jennifer T Kubota, Morgan G Seiler, and Elizabeth A Phelps. The effects of social context and acute stress on decision making under uncertainty. Psychological science, 26(12):1918-1926, 2015.

[14] Thackery I Brown, Stephanie A Gagnon, and Anthony D Wagner. Stress disrupts human hippocampal-prefrontal function during prospective spatial navigation and hinders flexible behavior. Current Biology, 30(10), 2020.

[15] M Fechir, T Schlereth, T Purat, S Kritzmann, C Geber, T Eberle, M Gamer, and F Birklein. Patterns of sympathetic responses induced by different stress tasks. The open neurology journal, 2:25, 2008.

[16] Ernst Roidl, Berit Frehse, and Rainer Höger. Emotional states of drivers and the impact on speed, acceleration and traffic violations-a simulator study. Accident Analysis \& Prevention, 70:282-292, 2014.

[17] Mara Mather, Marissa A Gorlick, and Nichole R Lighthall. To brake or accelerate when the light turns yellow? stress reduces older adults' risk taking in a driving game. Psychological science, 20(2):174-176, 2009.

[18] GM Hancock, PA Hancock, and CM Janelle. The impact of emotions and predominant emotion regulation technique on driving performance. Work 41(Supplement 1):3608-3611, 2012.

[19] Jesse Michaels, Romain Chaumillon, David Nguyen-Tri, Donald Watanabe, Pierro Hirsch, Francois Bellavance, Guillaume Giraudet, Delphine Bernardin, and Jocelyn Faubert. Driving simulator scenarios and measures to faithfully evaluate risky driving behavior: A comparative study of different driver age groups. PLOS ONE, 12:1-24, 102017.

[20] Gerald Matthews and Paula A Desmond. Stress and driving performance: Implications for design and training. Lawrence Erlbaum Associates Publishers, Mahwah, N.J, 2001.

[21] Hillary Abraham, Chaiwoo Lee, Samantha Brady, Craig Fitzgerald, Bruce Mehler, Bryan Reimer, and Joseph F Coughlin. Autonomous vehicles and alternatives to driving: trust, preferences, and effects of age. In Proceedings of the Transportation Research Board 96th Annual Meeting (TRB'17), 2017.

[22] Ericka Rovira, Anne Collins McLaughlin, Richard Pak, and Luke High. Looking for age differences in self-driving vehicles: Examining the effects of automation reliability, driving risk, and physical impairment on trust. Frontiers in Psychology, 10:800, 2019

[23] Masato Gokan, Daisuke Yamaguchi, and Toshiya Hirose. Driving characteristics when autonomous driving change to driver in low alertness and awake from sleeping. In WCX World Congress Experience. SAE International, apr 2018.

[24] Na Du, Feng Zhou, Elizabeth M. Pulver, Dawn M. Tilbury, Lionel P. Robert, Anuj K. Pradhan, and X. Jessie Yang. Examining the effects of emotional valence and arousal on takeover performance in conditionally automated driving Transportation Research Part C: Emerging Technologies, 112:78 - 87, 2020.

[25] Hua Cai and Yingzi Lin. Modeling of operators' emotion and task performance in a virtual driving environment. International fournal of Human-Computer Studies, 69(9):571-586, 2011.

[26] Joseph F Coughlin, Bryan Reimer, and Bruce Mehler. Monitoring, managing, and motivating driver safety and well-being. IEEE Pervasive Computing, 10(3):14-21, 2011.

[27] J SAE. 3016-2018, taxonomy and definitions for terms related to driving automation systems for on-road motor vehicles. Society of Automobile Engineers, sae. org, 2018.

[28] Dacher Keltner. Signs of appeasement: Evidence for the distinct displays of embarrassment, amusement, and shame. Journal of personality and social psychology, 68(3):441, 1995.

[29] James A Russell. A circumplex model of affect. Fournal of personality and social psychology, 39(6):1161, 1980.

[30] Vanessa L Castro, Yanhua Cheng, Amy G Halberstadt, and Daniel Grühn. Eureka! a conceptual model of emotion understanding. Emotion Review, 8(3):258-268, 2016.
[31] Javier Hernandez, Rob R Morris, and Rosalind W Picard. Call center stress recognition with person-specific models. In International Conference on Affective Computing and Intelligent Interaction, pages 125-134. Springer, 2011.

[32] Allen L Edwards. The relationship between the judged desirability of a trait and the probability that the trait will be endorsed. Fournal of Applied Psychology, 37(2):90, 1953.

[33] Delroy L Paulhus. Two-component models of socially desirable responding. fournal of personality and social psychology, 46(3):598, 1984.

[34] Karim S Kassam and Wendy Berry Mendes. The effects of measuring emotion: Physiological reactions to emotional situations depend on whether someone is asking. PloS one, 8(6), 2013.

[35] Peter Kuppens. It's about time: A special section on affect dynamics. Emotion Review, 7(4):297-300, 2015.

[36] Klaus RR Scherer. Towards a prediction and data driven computational process model of emotion. IEEE Transactions on Affective Computing, 2019.

[37] Sylvia D Kreibig. Autonomic nervous system activity in emotion: A review. Biological psychology, 84(3):394-421, 2010.

[38] Bruce Mehler, Bryan Reimer, and Joseph F. Coughlin. Physiological reactivity to graded levels of cognitive workload across three age groups: An on-road evaluation. Proceedings of the Human Factors and Ergonomics Society Annual Meeting, 54(24):2062-2066, 2010.

[39] Stephen H Fairclough, Marjolein van der Zwaag, Elena Spiridon, and Joyce Westerink. Effects of mood induction via music on cardiovascular measures of negative emotion during simulated driving. Physiology \& behavior, 129:173-180, 2014.

[40] William M Suess, A Barney Alexander, Deborah D Smith, Helga W Sweeney, and Richard J Marion. The effects of psychological stress on respiration: a preliminary study of anxiety and hyperventilation. Psychophysiology, 17(6):535$540,1980$.

[41] Yuri Masaoka and Ikuo Homma. Anxiety and respiratory patterns: their relationship during mental stress and physical load. International fournal of Psychophysiology, 27(2):153-159, 1997.

[42] Elke Vlemincx, Joachim Taelman, Steven De Peuter, Ilse Van Diest, and Omer Van Den Bergh. Sigh rate and respiratory variability during mental load and sustained attention. Psychophysiology, 48(1):117-120, 2011.

[43] Jennifer A Healey and Rosalind W Picard. Detecting stress during real-world driving tasks using physiological sensors. IEEE Transactions on intelligent transportation systems, 6(2):156-166, 2005.

[44] J. S. Khai Ooi, S. A. Ahmad, Y. Z. Chong, S. H. Md Ali, G. Ai, and H. Wagatsuma. Driver emotion recognition framework based on electrodermal activity measurements during simulated driving conditions. In 2016 IEEE EMBS Conference on Biomedical Engineering and Sciences (IECBES), pages 365-369, Kuala Lumpur, Malaysia, 2016. IEEE.

[45] H. Sawai, G. Inou, and E. Koyama. Evaluating optimal arousal level during the task based on performance and positive mood: Extracting indices reflecting the relationship among arousal, performance, and mood. In 2015 3rd International Conference on Applied Computing and Information Technology/2nd International Conference on Computational Science and Intelligence, pages 213-218, 2015.

[46] Yoko Nagai, Christopher Iain Jones, and Arjune Sen. Galvanic skin response (gsr)/electrodermal/skin conductance biofeedback on epilepsy: a systematic review and meta-analysis. Frontiers in neurology, 10:377, 2019.

[47] Bryan Reimer and Bruce Mehler. The impact of cognitive workload on physiological arousal in young adult drivers: a field study and simulation validation. Ergonomics, 54(10):932-942, 2011.

[48] C Ramon, A Clarion, Claudine Gehin, C Petit, Christian Collet, and André Dittmar. An integrated platform to assess driver's physiological and functional states. Conference proceedings : ... Annual International Conference of the IEEE Engineering in Medicine and Biology Society. IEEE Engineering in Medicine and Biology Society. Conference, 2008:506-9, 022008.

[49] Evangelos Paschalidis, Charisma F Choudhury, and Stephane Hess. Combining driving simulator and physiological sensor data in a latent variable model to incorporate the effect of stress in car-following behaviour. Analytic methods in accident research, 22:100089, 2019.

[50] VL Schechtman, KA Kluge, and RM Harper. Time-domain system for assessing variation in heart rate. Medical and Biological Engineering and Computing, 26(4):367-373, 1988.

[51] Charles J Peek. A primer of biofeedback instrumentation. The Guilford Press, 1995.

[52] Gregory A Miller. Mistreating psychology in the decades of the brain. Perspectives on Psychological Science, 5(6):716-743, 2010.

[53] Erika H Siegel, Molly K Sands, Wim Van den Noortgate, Paul Condon, Yale Chang, Jennifer Dy, Karen S Quigley, and Lisa Feldman Barrett. Emotion fingerprints or emotion populations? a meta-analytic investigation of autonomic features of emotion categories. Psychological bulletin, 144(4):343, 2018.

[54] Christine Spencer, Daniel Moore, Gary McKeown, Lucy Rutherford, and Gawain Morrison. Context matters: protocol ordering effects on physiological arousal and experienced stress during a simulated driving task. In 2019 8th International Conference on Affective Computing and Intelligent Interaction (ACII), pages 1-7, 
Cambridge, UK, 2019. IEEE, Association for Computing Machinery.

[55] Hua Gao, Anil Yüce, and Jean-Philippe Thiran. Detecting emotional stress from facial expressions for driving safety. In 2014 IEEE International Conference on Image Processing (ICIP), pages 5961-5965. IEEE, 2014.

[56] G Giannakakis, Matthew Pediaditis, Dimitris Manousos, Eleni Kazantzaki, Franco Chiarugi, Panagiotis G Simos, Kostas Marias, and Manolis Tsiknakis. Stress and anxiety detection using facial cues from videos. Biomedical Signal Processing and Control, 31:89-101, 2017.

[57] Carlos Crivelli and Alan J Fridlund. Facial displays are tools for social influence. Trends in Cognitive Sciences, 22(5):388-399, 2018.

[58] Maria Gendron, Carlos Crivelli, and Lisa Feldman Barrett. Universality reconsidered: Diversity in making meaning of facial expressions. Current directions in psychological science, 27(4):211-219, 2018.

[59] Lisa Feldman Barrett, Ralph Adolphs, Stacy Marsella, Aleix M Martinez, and Seth D Pollak. Emotional expressions reconsidered: challenges to inferring emotion from human facial movements. Psychological Science in the Public Interest, 20(1):1-68, 2019.

[60] Judee K Burgoon and Beth A Le Poire. Nonverbal cues and interpersonal judgments: Participant and observer perceptions of intimacy, dominance, composure, and formality. Communications Monographs, 66(2):105-124, 1999.

[61] Katharine H Greenaway, Elise K Kalokerinos, and Lisa A Williams. Context is everything (in emotion research). Social and Personality Psychology Compass, 12(6):e12393, 2018

[62] Magdalena Rychlowska, Rachael E Jack, Oliver GB Garrod, Philippe G Schyns, Jared D Martin, and Paula M Niedenthal. Functional smiles: Tools for love, sympathy, and war. Psychological science, 28(9):1259-1270, 2017.

[63] Julie A Woodzicka and Marianne LaFrance. Real versus imagined gender harassment. Fournal of Social Issues, 57(1):15-30, 2001.

[64] Ronald E Riggio and Howard S Friedman. Impression formation: The role of expressive behavior. Fournal of Personality and Social Psychology, 50(2):421, 1986.

[65] Nancy J Briton and Judith A Hall. Beliefs about female and male nonverbal communication. Sex Roles, 32(1-2):79-90, 1995.

[66] Ann M Kring and Albert H Gordon. Sex differences in emotion: expression, ex perience, and physiology. Journal of personality and social psychology, 74(3):686, 1998.

[67] Agneta Fischer and Marianne LaFrance. What Drives the Smile and the Tear: Why Women Are More Emotionally Expressive Than Men. Emotion Review, 7(1):22-29, jan 2015.

[68] Marianne LaFrance, Marvin A Hecht, and Elizabeth Levy Paluck. The contingent smile: a meta-analysis of sex differences in smiling. Psychological bulletin, 129(2):305, 2003.

[69] A. J.; Fridlund. Human Facial Expression: An Evolutionary View. Academic Press, Academic Press, 1994.

[70] Soujanya Poria, Erik Cambria, Rajiv Bajpai, and Amir Hussain. A review of affective computing: From unimodal analysis to multimodal fusion. Information Fusion, 37:98-125, 2017

[71] Simone Bianco, Paolo Napoletano, and Raimondo Schettini. Multimodal car driver stress recognition. In Proceedings of the 13th EAI International Conference on Pervasive Computing Technologies for Healthcare, pages 302-307, 2019.

[72] Martin Magdin, Michal Kohútek, Štefan Koprda, and Zoltán Balogh. Emosens the proposal of system for recognition of emotion with sdk affectiva and various sensors. In De-Shuang Huang, Vitoantonio Bevilacqua, and Prashan Premaratne, editors, Intelligent Computing Theories and Application, pages 400-411, Cham, 2019. Springer International Publishing.

[73] Sidney K D'mello and Jacqueline Kory. A review and meta-analysis of multimodal affect detection systems. ACM Computing Surveys (CSUR), 47(3):1-36 2015.

[74] Carlos Crivelli and Alan J. Fridlund. Facial displays are tools for social influence. Trends in Cognitive Sciences, 22(5):388 - 399, 2018

[75] Yan Liu, Shai H Zhu, Guo H Wang, Fei Ye, and Peng Z Li. Validity and reliability of multiparameter physiological measurements recorded by the equivital lifemonitor during activities of various intensities. Journal of occupational and environmental hygiene, 10(2):78-85, 2013

[76] William J Tharion, Mark J Buller, Adam W Potter, Anthony J Karis, Victoria Goetz, and Reed W Hoyt. Acceptability and usability of an ambulatory health monitoring system for use by military personnel. IIE Transactions on Occupational Ergonomics and Human Factors, 1(4):203-214, 2013.

[77] Abimbola A Akintola, Vera van de Pol, Daniel Bimmel, Arie C Maan, and Diana van Heemst. Comparative analysis of the equivital eq02 lifemonitor with holter ambulatory ecg device for continuous measurement of ecg, heart rate, and heart rate variability: A validation study for precision and accuracy. Frontiers in physiology, 7:391, 2016.

[78] SHORE. Emotion recognition software shore ${ }^{\circledR}$ : Fast, reliable and real-time capable. http://www.iis.fraunhofer.de/shore, 2020.

[79] Sensum. Sensum empathic ai developer kit. https://sensum.co/, January 2020.
[80] Gareth James, Daniela Witten, Trevor Hastie, and Robert Tibshirani. An introduction to statistical learning, volume 112. Springer, 2013.

[81] R Core Team. R: A Language and Environment for Statistical Computing. R Foundation for Statistical Computing, Vienna, Austria, 2020.

[82] Simon N Wood. Fast stable restricted maximum likelihood and marginal likelihood estimation of semiparametric generalized linear models. Fournal of the Royal Statistical Society: Series B (Statistical Methodology), 73(1):3-36, 2011.

[83] Alain Zuur, Elena N Ieno, Neil Walker, Anatoly A Saveliev, and Graham M Smith. Mixed effects models and extensions in ecology with $R$. Springer Science \& Business Media, 2009

[84] Hadley Wickham. ggplot2: Elegant Graphics for Data Analysis. Springer-Verlag New York, 2016.

[85] Stefano Coretta. tidymv: Tidy Model Visualisation for Generalised Additive Models, 2020. R package version 3.0.0.

[86] Wolfram Boucsein. Electrodermal activity. Springer Science \& Business Media, 2012.

[87] Arvid Kappas and J Descôteaux. Of butterflies and roaring thunder: Nonverbal communication in interaction and regulation of emotion. In Nonverbal behavior in clinical settings, number November, pages 45-74. Oxford University Press, New York, 2003

[88] Suzanne Pieper, Jos F Brosschot, Rien van der Leeden, and Julian F Thayer. Prolonged cardiac effects of momentary assessed stressful events and worry episodes. Psychosomatic Medicine, 2010.

[89] Katherine A Herborn, James L Graves, Paul Jerem, Neil P Evans, Ruedi Nager, Dominic J McCafferty, and Dorothy EF McKeegan. Skin temperature reveals the intensity of acute stress. Physiology \& behavior, 152:225-230, 2015.

[90] Nadia Bianchi-Berthouze and Andrea Kleinsmith. A categorical approach to affective gesture recognition. Connection science, 15(4):259-269, 2003.

[91] Asha Kapur, Ajay Kapur, Naznin Virji-Babul, George Tzanetakis, and Peter F Driessen. Gesture-based affective computing on motion capture data. In International conference on affective computing and intelligent interaction, pages 1-7. Springer, 2005.

[92] Iain R Murray and John L Arnott. Toward the simulation of emotion in synthetic speech: A review of the literature on human vocal emotion. The fournal of the Acoustical Society of America, 93(2):1097-1108, 1993.

[93] Samuel Albanie, Arsha Nagrani, Andrea Vedaldi, and Andrew Zisserman. Emotion recognition in speech using cross-modal transfer in the wild. In Proceedings of the 26th ACM international conference on Multimedia, pages 292-301, 2018.

[94] Panagiotis Tzirakis, Jiehao Zhang, and Bjorn W Schuller. End-to-end speech emotion recognition using deep neural networks. In 2018 IEEE International Conference on Acoustics, Speech and Signal Processing (ICASSP), pages 5089-5093. IEEE, 2018.

[95] Linda Ng Boyle and Fred Mannering. Impact of traveler advisory systems on driving speed: some new evidence. Transportation Research Part C: Emerging Technologies, 12(1):57-72, 2004.

[96] Birsen Donmez, Linda Ng Boyle, and John D Lee. The impact of distraction mitigation strategies on driving performance. Human factors, 48(4):785-804, 2006.

[97] Shan Bao and Linda Ng Boyle. Driver performance at two-way stop-controlled intersections on divided highways. Transportation Research Record, 2069(1):2632, 2008.

[98] Sebastian Zepf, Monique Dittrich, Javier Hernandez, and Alexander Schmitt. Towards empathetic car interfaces: Emotional triggers while driving. In Extended Abstracts of the 2019 CHI Conference on Human Factors in Computing Systems, pages 1-6, 2019 .

[99] Hynek Bořil, Pinar Boyraz, and John HL Hansen. Towards multimodal driver's stress detection. In Digital signal processing for in-vehicle systems and safety, pages 3-19. Springer, 2012.

[100] Hui-Sup Cho, Young-Jin Park, Hong-Kun Lyu, and Jin-Ho Cho. Novel heart rate detection method using uwb impulse radar. fournal of Signal Processing Systems, 87(2):229-239, 2017.

[101] Gerard De Haan and Vincent Jeanne. Robust pulse rate from chrominance-based rppg. IEEE Transactions on Biomedical Engineering, 60(10):2878-2886, 2013.

[102] Guha Balakrishnan, Fredo Durand, and John Guttag. Detecting pulse from head motions in video. In Proceedings of the IEEE Conference on Computer Vision and Pattern Recognition, pages 3430-3437, 2013.

[103] Ming-Zher Poh, Daniel J McDuff, and Rosalind W Picard. Advancements in noncontact, multiparameter physiological measurements using a webcam. IEEE transactions on biomedical engineering, 58(1):7-11, 2010.

[104] Daniel McDuff, Sarah Gontarek, and Rosalind W Picard. Improvements in remote cardiopulmonary measurement using a five band digital camera. IEEE Transactions on Biomedical Engineering, 61(10):2593-2601, 2014.

[105] Ali Al-Naji and Javaan Chahl. Simultaneous tracking of cardiorespiratory signals for multiple persons using a machine vision system with noise artifact removal. IEEE journal of translational engineering in health and medicine, 5:1-10, 2017.

[106] Javier Hernandez, Pablo Paredes, Asta Roseway, and Mary Czerwinski. Under pressure: Sensing stress of computing users. In In Proc. of Computer and Human 
Interaction. Citeseer, 2014. 